\title{
Bốn trường đại học có tổng số công bố của các nhà khoa học vượt 1000
}

\author{
Nguyễn Minh Hoàng \\ Nghiên cứu sinh \\ Ritsumeikan Asia Pacific University \\ Address:1-1 Jumonjibaru, Beppu, Oita 874-8577 Japan

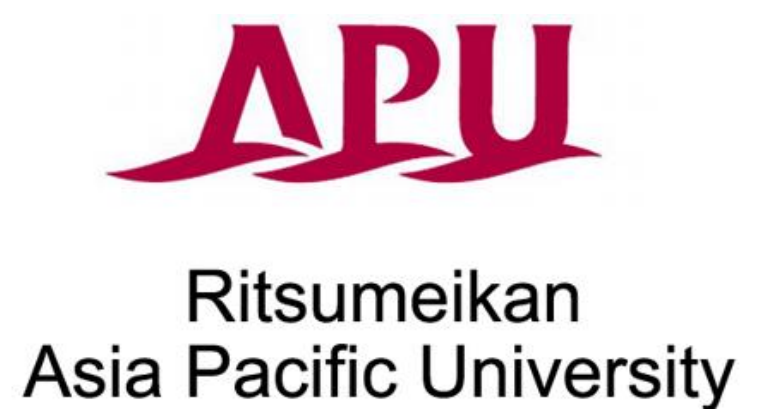

Ngày 13 tháng 6 năm 2021

Theo dữ liệu Publons (ISI Web of Science), có bốn trường Đại học Việt Nam có tổng số công bố khoa học của các nhà khoa học vượt 1000 và 14 nhà nghiên cứu người Việt tại các cơ sở nghiên cứu trong nước có trên 100 công bố khoa học.

Trong thời gian vừa qua, các cơ sở nghiên cứu khoa học đã có đóng góp to lớn vào sự phát triển nhanh chóng của khoa học và kỹ thuật tại Việt Nam. Để tìm hiểu và đánh giá năng suất khoa học của các cơ sở nghiên cứu trên cả nước và các nhà khoa học nổi bậc, cơ sở dữ liệu (CSDL) Publons là một trong những nguồn dữ liệu đáng tin cậy. Sau khi liên kết với Clarivate Web of Science, các bản xếp hạng nghiên cứu trên Publons đều chỉ dùng các công bố khoa học được xuất bản trên các tạp chí được chỉ mục trong CSDL Web of Science (WOS vẫn thường được biết đến ở Việt Nam với cái tên ISI - Institute for Scientific Information). Một phần dữ liệu WOS này được mở miễn phí cho người sử dụng Internet qua https://publons.com. 
Tính tới thời điểm hiện tại (13/06/2021), trên CSDL Publons, có bốn cơ sở nghiên cứu tại Việt Nam có tổng số công bố khoa học quốc tế uy tín của các nhà khoa học vượt 1000. Cụ thể là trường Đại học Tôn Đức Thắng (8166 công bố), trường Đại học Duy Tân (2166 công bố), trường Đại học Phenikaa (1935 công bố), và trường Đại học Bách Khoa - Đại học Quốc gia TP.HCM (1006 công bố). Có thể thấy số lượng công bố của trường Đại học Tôn Đức Thắng là rất ấn tượng với số lượng nghiên cứu gần gấp bốn lần trường Đại học Duy Tân xếp ở vị trí thứ 2 (xem hình 1).

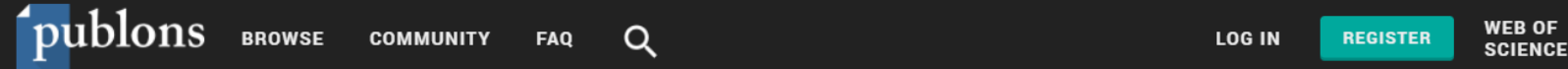

Home , Institutions , Institution Leaderboard

\section{Institutions}

\begin{tabular}{|c|c|c|c|c|c|c|}
\hline HIDE FILTERS & CLEAR ALL & & & & & \\
\hline \multicolumn{2}{|c|}{ Institution Name } & & \multicolumn{4}{|l|}{$\begin{array}{l}\text { Country/Region } \\
\text { Vietnam }\end{array}$} \\
\hline \multicolumn{3}{|l|}{93 results } & & \multicolumn{3}{|c|}{ Last updated 1 hour, 48 minutes ago } \\
\hline \# & INSTITUTION & RESEARCHERS & $\begin{array}{r}\text { TOP } \\
\text { REVIEWERS }\end{array}$ & $\begin{array}{l}\text { VERIFIED } \\
\text { REVIEWS }\end{array}$ & $\begin{array}{r}\text { VERIFIED } \\
\text { REVIEWS LAST } 12 \\
\text { MONTHS }\end{array}$ & $\begin{array}{r}\text { CORE } \\
\text { COLLECTION } \\
\text { PUBLICATIONS }\end{array}$ \\
\hline $753 r d$ & Ton Duc Thang University & 262 & 13 & 4.386 & 1.381 & 8.166 \\
\hline 2080th & Duy Tan University & 57 & 5 & 3.321 & 1.290 & 2.166 \\
\hline 2271 st & Phenikaa University & 77 & 5 & 1.392 & 377 & 1.937 \\
\hline 3635th & Ho Chi Minh City University of Technology & 95 & 3 & 1.052 & 316 & 1.006 \\
\hline 4939th & Hanoi University of Science and Technology & 85 & 2 & 785 & 254 & 643 \\
\hline
\end{tabular}

Hình 1: Bốn trường Đại học Việt Nam có tổng số công bố khoa học của các nhà nghiên cứu vượt 1000

Tổng số công bố khoa học của các trường Đại học nói trên phụ thuộc nhiều vào khả năng nghiên cứu của một số nhà khoa học nổi bậc đang công tác tại trường. Tính tới thời điểm hiện tại, có 14 nhà khoa học có trên 100 công bố được ghi nhận trên CSDL Publons. Riêng các nhà khoa học có trên 100 công bố tại trường Đại học Tôn Đức Thắng, trường Đại học Phenikaa, và trường Trường Đại học Bách Khoa - Đại học Quốc gia TP.HCM đã đóng góp hơn một nửa (57.14\%) (xem hình 2). 


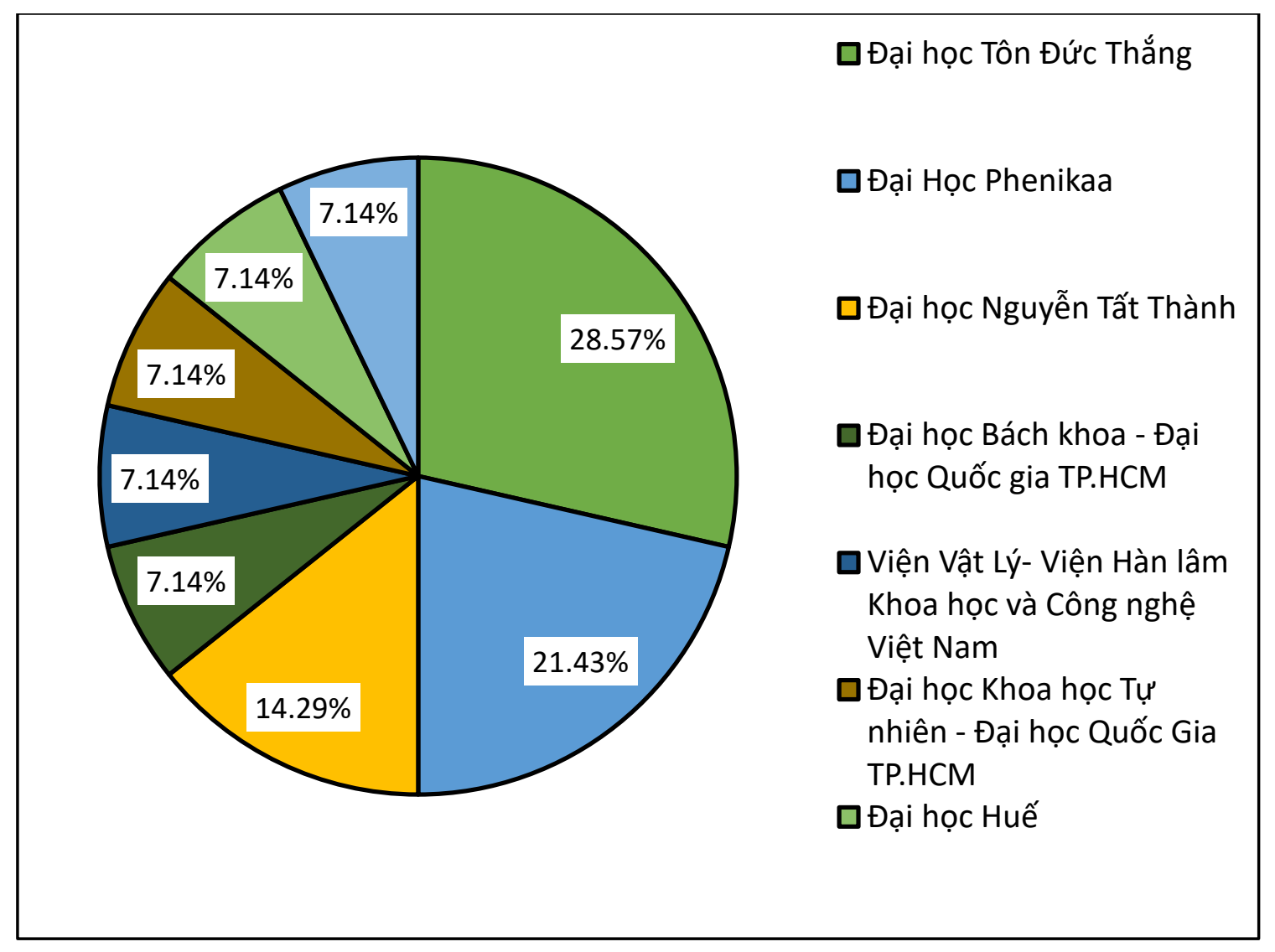

Hình 2: Tỷ lệ các nhà khoa học có trên 100 công bố theo đơn vị công tác

Trong ba trường, trường Đại học Tôn Đức Thắng đóng góp nhiều nhất với bốn nhà nghiên cứu nổi bậc: PGS.TS. Nguyễn Thời Trung, TS. Nguyễn Huy Tuấn, TS. Đỗ Đình Thuấn, và TS. Nguyễn Trương Khang. Tiếp đó là trường Đai Học Phenikaa với ba nhà nghiên cứu: GS.TS. Nguyễn Văn Hiếu, TS. Vương Quân Hoàng, và TS. Đào Văn Dương. Sau cùng là trường Đại học Bách Khoa - Đại học Quốc gia TP.HCM với sự góp mặt của nhà nghiên cứu GS.TS Nguyễn Xuân Hùng (xem bảng 1).

Bảng 1: Danh sách các nhà khoa học Việt Nam có trên 100 công bố khoa học

\begin{tabular}{|c|c|c|c|c|}
\hline $\begin{array}{c}\text { Thứ } \\
\text { tự }\end{array}$ & Nhà khoa học & Cơ sở nghiên cứu & $\begin{array}{c}\text { Số lượng } \\
\text { công bố }\end{array}$ & $\begin{array}{c}\text { Nhóm } \\
\text { ngành }\end{array}$ \\
\hline 1 & Nguyễn Thời Trung & Đại học Tôn Đức Thắng & 256 & KHTN \\
\hline 2 & Võ Nguyễn Đại Việt & Đại học Nguyễn Tất Thành & 238 & KHTN \\
\hline 3 & Nguyễn Xuân Hùng & Đại học Bách khoa - ĐHQG TP.HCM & 211 & KHTN \\
\hline 4 & Nguyễn Bá Ân & $\begin{array}{c}\text { Viện Vật Lý- Viện Hàn lâm Khoa } \\
\text { học và Công nghệ Việt Nam }\end{array}$ & 156 & KHTN \\
\hline 5 & Nguyễn Huy Tuấn & Đại học Tôn Đức Thắng & 153 & KHTN \\
\hline
\end{tabular}




\begin{tabular}{|c|c|c|c|c|}
\hline 6 & Phạm Văn Phúc & $\begin{array}{c}\text { Đại học Khoa học Tự nhiên - ĐHQG } \\
\text { TP.HCM }\end{array}$ & 151 & KHTN \\
\hline 7 & Đỗ Đình Thuấn & Đại học Tôn Đức Thắng & 139 & KHTN \\
\hline 8 & Nguyễn Văn Hiếu & Đại Học Phenikaa & 131 & KHTN \\
\hline 9 & Nguyễn Duy Trinh & Đại học Nguyễn Tất Thành & 113 & KHTN \\
\hline 10 & Vương Quân Hoàng & Đại Học Phenikaa & 103 & KHXH\&NV \\
\hline 11 & Đào Văn Dương & Đại Học Phenikaa & 102 & KHTN \\
\hline 12 & Hoàng Hữu Hạnh & Đại học Huế & 101 & KHTN \\
\hline 13 & Nguyễn Trương Khang & Đại học Tôn Đức Thắng & 100 & KHTN \\
\hline 14 & Chu Đình Tới & Đại học Sư phạm Hà Nội & 100 & KHTN \\
\hline
\end{tabular}

(*Nguồn: Dũ liệu trích xuất ngày 13-6-2021; https://publons.com)

Đáng nói, mặc dù còn rất trẻ, Đại học Phenikaa không những nằm trong bốn cơ sở nghiên cứu có trên 1000 công bố khoa học mà còn lại là nơi làm công tác của ba nhà nghiên cứu có trên 100 công bố khoa học, cao thứ nhì cả nước (21.43\%). Lý do phía sau kết quả này của trường Đại học Phenikaa có thể nhờ không gian và văn hóa khoa học đặc hữu của đơn vị [1].

\begin{tabular}{|c|c|c|c|c|c|c|c|}
\hline pu & 1015 & COMMUNITY & Q & & LOG IN & REGISTER & $\begin{array}{l}\text { NEB OF } \\
\text { SCIENCE }\end{array}$ \\
\hline \multicolumn{8}{|c|}{ Home, Researchers } \\
\hline \# & RESEARCHERS & & INSTITUTION & \# PUBLICATIONS & $\begin{array}{r}\text { \# VERIFIED } \\
\text { REVIEWS }\end{array}$ & $\begin{array}{r}\text { \# VERIFIED } \\
\text { EDITOR } \\
\text { RECORDS }\end{array}$ & \\
\hline 1 & Nguyen Van Hieu & & Phenikaa University & 131 & 85 & & - \\
\hline 2 & Quan-Hoang Vuong & 운 & Phenikaa University & 103 & 166 & & 55 \\
\hline 3 & Van-Duong Dao & $\stackrel{\circ}{N^{\prime}}$ & Phenikaa University & 102 & 311 & & 13 \\
\hline 4 & Viet-Thanh Pham & & Phenikaa University & 90 & 198 & & - \\
\hline 5 & Auy T Pham & & Phenikaa University & 81 & 5 & & - \\
\hline 6 & Duc-Tan Tran & & Phenikaa University & 57 & 54 & & - \\
\hline 7 & RD Raja Das & & Phenikaa University & 56 & - & & - \\
\hline 8 & 8. Tran Quang Huy & & Phenikaa University & 56 & 53 & & - \\
\hline 9 & HuuTung Nguyen & & Phenikaa University & 46 & 9 & & - \\
\hline 10 & A Phung Van Dong & & Phenikaa University & 45 & - & & - \\
\hline
\end{tabular}

Hình 3: Top 10 nhà khoa học có nhiều công bố nhất của trường Đại học Phenikaa 
Ngoài các trường Đại học có trên 1000 bài công bố quốc tế, một số trường Đại học khác cũng có các nhà khoa học nổi bật có trên 100 công bố khoa học, như: hai nhà nghiên cứu TS. Võ Nguyễn Đại Việt và TS. Nguyễn Duy Trinh của trường Đại học Nguyễn Tất Thành; nhà nghiên cứu PGS.TS. Nguyễn Bá Ân của Viện Vật Lý - Viện Hàn lâm Khoa học và Công Nghệ Việt Nam; nhà nghiên cứu PGS.TS. Phạm Văn Phúc của trường Đại học Khoa học Tự nhiên - Đại học Quốc Gia TP.HCM; nhà nghiên cứu PGS.TS Hoàng Hữu Hạnh của trường Đại học Huế.

Nhà nghiên cứu có số lương công bố khoa học cao nhất cả nước, theo ghi nhận của CSDL Publons, là PGS.TS Nguyễn Thời Trung (ĐH Tôn Đức Thắng), một trong 10 nhà khoa học Việt Nam có tên trong top các nhà khoa học được trích dẫn nhiều nhất thế giới [2]. Xếp thứ hai là TS. Võ Nguyễn Đại Việt (ĐH Nguyễn Tất Thành), người nhận được giải thưởng bình duyệt toàn cầu Global Peer Review Awards của Publons [3]. Xếp thứ ba là GS.TS. Nguyễn Xuân Hùng (ĐH Bách Khoa - Đại học Quốc gia TP.HCM), người đã 7 năm liên tiếp lọt vào top $1 \%$ các nhà khoa học có nhiều trích dẫn nhất thế giới [4].

Trong danh sách 14 nhà nghiên cứu, hầu hết đều thuộc lĩnh vực Khoa Học Tự Nhiên (KHTN), chỉ có duy nhất một nhà khoa học có trên 100 công bố quốc tế được ghi nhận trong lĩnh vực Khoa Học Xã Hội và Nhân Văn (KHXH\&NV) là TS. Vương Quân Hoàng (ĐH Phenikaa), đáng chú ý với những bài trên các ấn phẩm trên nature.com, như Nature, Nature Human Behaviour, Scientific Data, và Humanities and Social Sciences Communications [5-8].

* Ghi chú: Dữ liệu sử dụng trong bài viết được lấy thủ công từ CSDL Publons và nhiều nhà nghiên cứu Việt Nam chưa cập nhật đầy đủ dữ liệu cho trang cá nhân của mình, nên chắc chắn sẽ còn thiếu sót. Ngoài ra bản thân WOS cũng có độ trễ trong cập nhật các ấn phẩm mới xuất bản.

\section{References}

[1] Nguyen, T. T. H. (2021). Những người kiến tạo không gian và văn hóa khoa học Phenikaa. Kinh tế và Dự báo. URL: https://kinhtevadubao.vn/nhung-nguoi-kien-tao-khong-gian-va-vanhoa-khoa-hoc-phenikaa-17112.html

[2] Ha, A. (2020). Nhà khoa học Việt Nam được trích dẫn nhiều nhất thế giới. Thanh Niên. URL: https://thanhnien.vn/giao-duc/nha-khoa-hoc-viet-nam-duoc-trich-dan-nhieu-nhat-thegioi-1175478.html

[3] NTTU. (2019). Giảng viên ĐH Nguyễn Tất Thành lọt vào top 1\% các nhà bình duyệt từ Việt Nam. Đại Học Nguyễn Tất Thành. URL: https://ntt.edu.vn/web/tin-tuc/giang-vien-dhnguyen-tat-thanh-lot-vao-top-1-cac-nha-binh-duyet-tu-viet-nam

[4] Thanh, N. (2020). Giáo sư Nguyễn Xuân Hùng: Lần thứ bảy liên tiếp vào top $1 \%$ thế giới. Tia Sáng. URL: https://tiasang.com.vn/-doi-moi-sang-tao/Giao-su-Nguyen-Xuan-Hung-Lanthu-bay-lien-tiep-vao-top-1-the-gioi--26665 
[5] Vuong, Q. H. (2020). Reform retractions to make them more transparent. Nature, 582(7811), 149.

[6] Vuong, Q. H. (2018). The (ir)rational consideration of the cost of science in transition economies. Nature Human Behaviour, 2(1), 5.

[7] Vuong, Q. H., et al. (2018). An open database of productivity in Vietnam's social sciences and humanities for public use. Scientific Data, 5, 180188.

[8] Vuong, Q. H., et al. (2021). Assessing the ideological homogeneity in entrepreneurial finance research by highly cited publications. Humanities and Social Sciences Communications, 8 , 110. 